\title{
Proposal for a Wheel Suspension Mechanism with Controlled Characteristics
}

\author{
Jan Kovanda* \\ Czech Technical University in Prague, Faculty of Transportation Sciences \\ * Corresponding author: kovanda@lss.fd.cvut.cz
}

DOI: 10.2478/v10158-010-0016-y

\begin{abstract}
This article presents the design of an overconstrained suspension mechanism based on five-link suspension. The system contains kinematic joints with a simple on-off control system, which enables it to change the kinematic scheme and, consequently, the suspension characteristics (the longitudinal and lateral movement of the wheel centre point, steering angle, camber and caster angle, etc.). A wheel suspension mechanism with controlled characteristics enables the adaption of suspension behavior according to the driving situation and an optimization of the vehicle dynamics, handling and comfort.
\end{abstract}

KEY WORDS: vehicle suspension, suspension characteristics, redundant wheel suspension, five-link suspension, adaptive suspension kinematics

\section{INTRODUCTON}

Wheel suspension and control mechanism is a constrained mechanical system allowing the connection of a sprung mass (car body) and spring unsprung mass (wheel, wheel support, accessories) in such a way as to provide the best possible conditions for tyre-road contact and driving comfort. The position of the wheel on the road and the position of the key geometric elements during vertical movement (springing to car body) is described by charts known as suspension characteristics. Their forms are based on the required tyre-to-road position persistence (this condition is derived from the design of the tyre used) drive comfort affected by the transmission of vibration, forces transmission onto car body during braking, acceleration, and direction change. These characteristics can often be a compromised solution attained from the various requirements of driving safety and comfort conditions. The requirement of a forward incline during braking is, for example, contradictory with the vibration transmission when driving along uneven roads. Moreover, the definition of these characteristics are formed based on the predictable states of the vehicle and are not adaptive according to changes in conditions or the requirements of the current driving conditions of the vehicle.

\section{SOLUTION}

The presented solution is based on the presumption of mechatronic features existing in the vehicle, i.e., the action elements that set the requested state of the mechanism and sensors for the driving requirements detection. The wheel suspension mechanism is, in this case, constructed overconstrained (members and kinematic pairs) than needed to reach 
one degree of freedom for vertical movement (caused by springing), and some kinematic pairs must, therefore, be actively controlled. Without this, the system would be kinematically overstated and would have zero degrees of freedom and redundant constraint equations in case of blocking more of the controlled kinematic pairs than requested to reach the needed mobility. On the other hand, more free pairs would lead to unwanted mobility. Therefore, the system is equipped with pairs that can be blocked or activated by the control system of the mechanism in order to reach under this setting the requested one degree of freedom. The chosen kinematic pairs can be blocked or activated by the control system in such a way that the suspension has the required characteristics, or the rigidity of the springing-suspension members in the kinematic pairs and the elasto-kinematics of the suspension is affected, or it can fluctuate between two extreme characteristics of suspension.

This kinematics construction, equipped with the control of the mechanism, makes the suspension designing process more complicated, and it brings with it active members and potentially increases unsprung masses. However, it even brings the possibility to optimally choose the suspension characteristics improving the tyre-road contact, horizontal, vertical, and lateral dynamics of the vehicle and affects the noise and vibrations in the vehicle. By this means, it even improves the safety and driving comfort.

It is necessary to say the kinematically pre-set systems are used in cars already. As examples, we can state the chasis with compound crank axle and exquisite elastokinematic characteristics.

\section{EXAMPLE OF SOLUTION}

This system can be described by a suspension system with five elements (Figure 1), without the steering mechanism (rear axle). There is one element added in the suspension it is the binary member with two spherical pairs. The mechanism would become a rigid (zero degrees of freedom) construction and therefore we locate two selected spherical pairs on the chassis of the vehicle in such a way that their sliding movement is allowed and by active power members we will control their movement (released-blocked status). The result will get the different kinematic schemes for different settings that end up in different suspension characteristics. In the example mentioned above (transmission of vibration and forward inclination during braking), the longitudinal wheel movement characteristics is principal (Figure 3). This longitudinal movement of the wheel centre can either support the contra-forward inclining movement during braking (the wheel travels forward and upward when braking) or we can limit the transmission of vibration when braking on uneven surface (the wheel travels backward and the stroke power is limited when getting on the terrain obstacle). The solution made by blocking and releasing the respective pair (Figure 2 and 3) can affect the behaviour described in accordance with other input data, which comes to the control systems, and we can choose the optimal settings for the respective setting (speed, intensity of braking, road surface quality). There are various situations applicable on lateral and sideway dynamics (e.g., the affection of mutual co-action and relation of wheel on the same axis when driving through the curve under different conditions, etc.). The properties of many suspension types can be derived from their kinematic schemes of five-element suspension, the given solution can be taken as general, and the principle can be used for all suspension types and steering settings in road vehicles of all categories, air vehicle carriages, etc. 


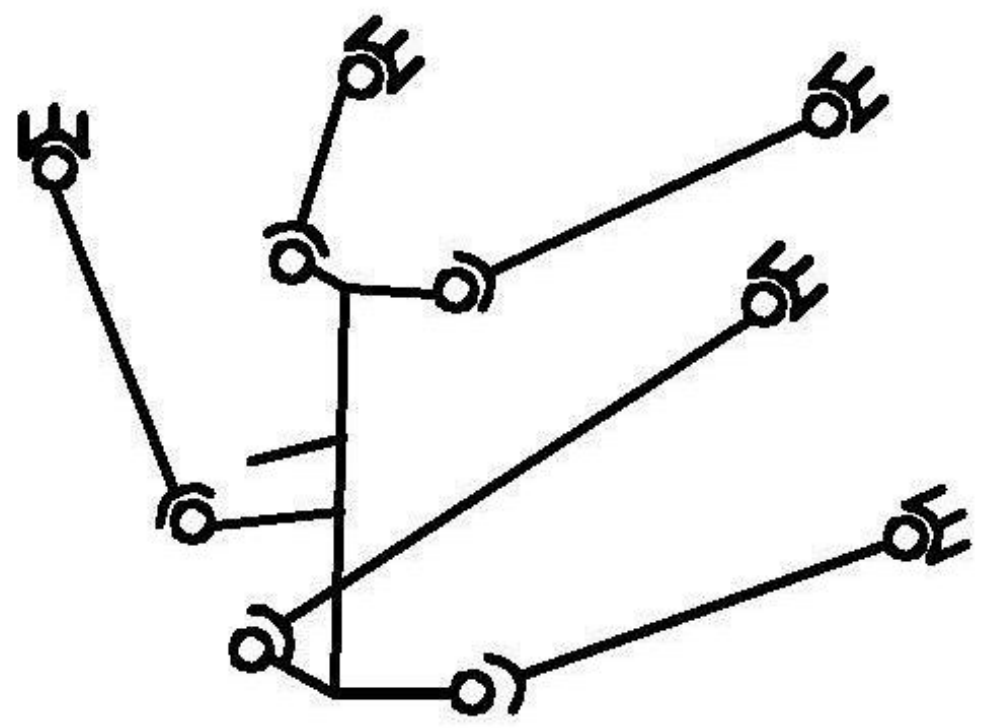

Figure 1: Original scheme of five-element suspension with fixed kinematic scheme.

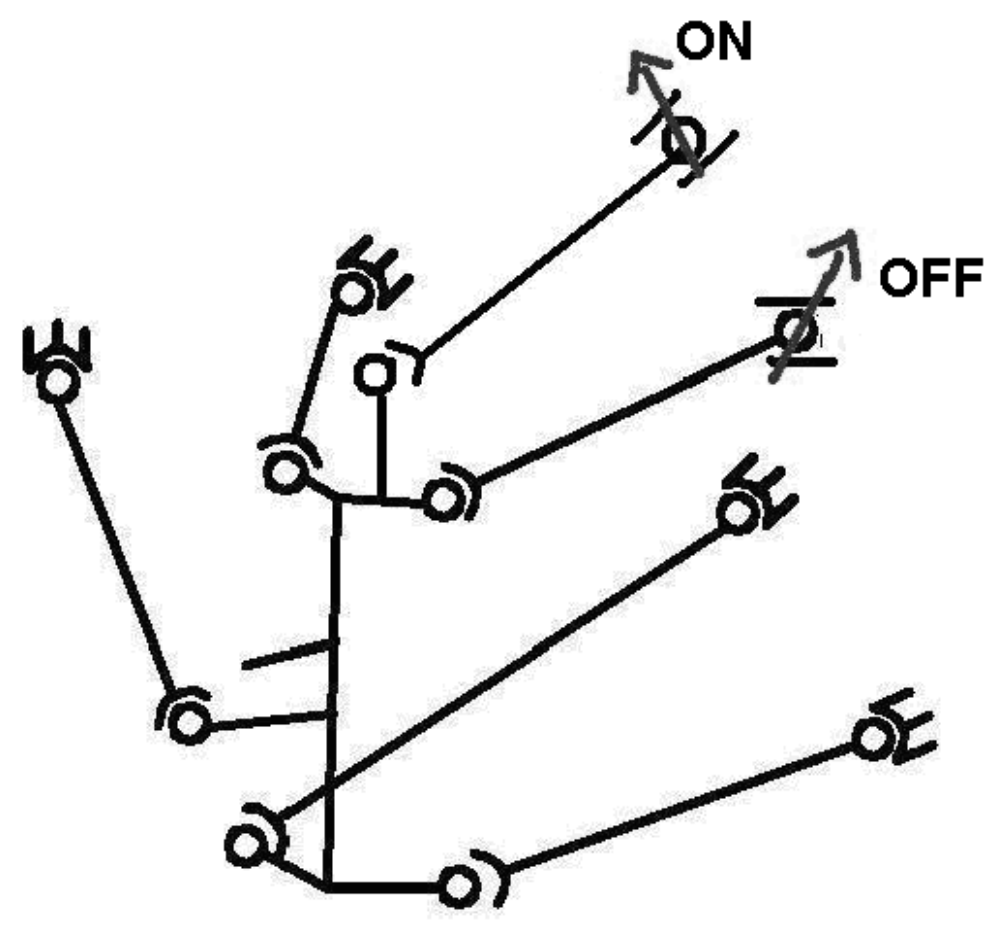




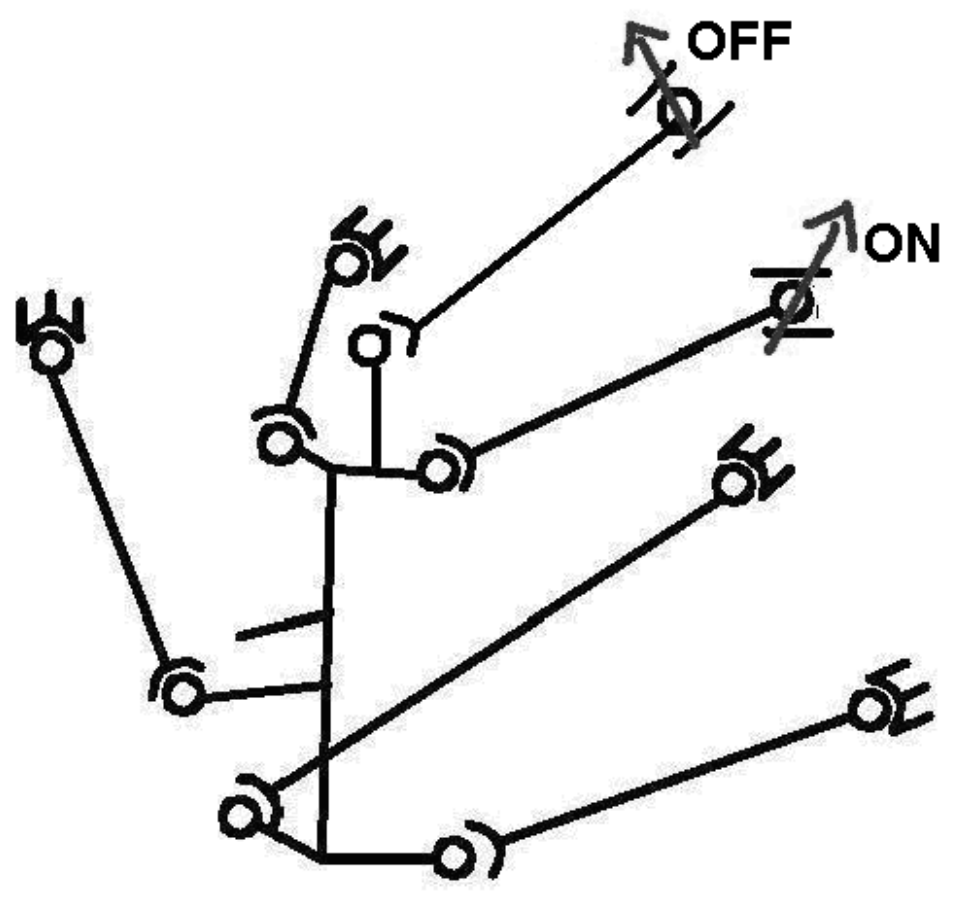

Figure 2: Modified suspension with additional kinematic constraint and sliding linkage of spherical joints with shift management (indicated by arrows, OFF is unlocked and sliding).

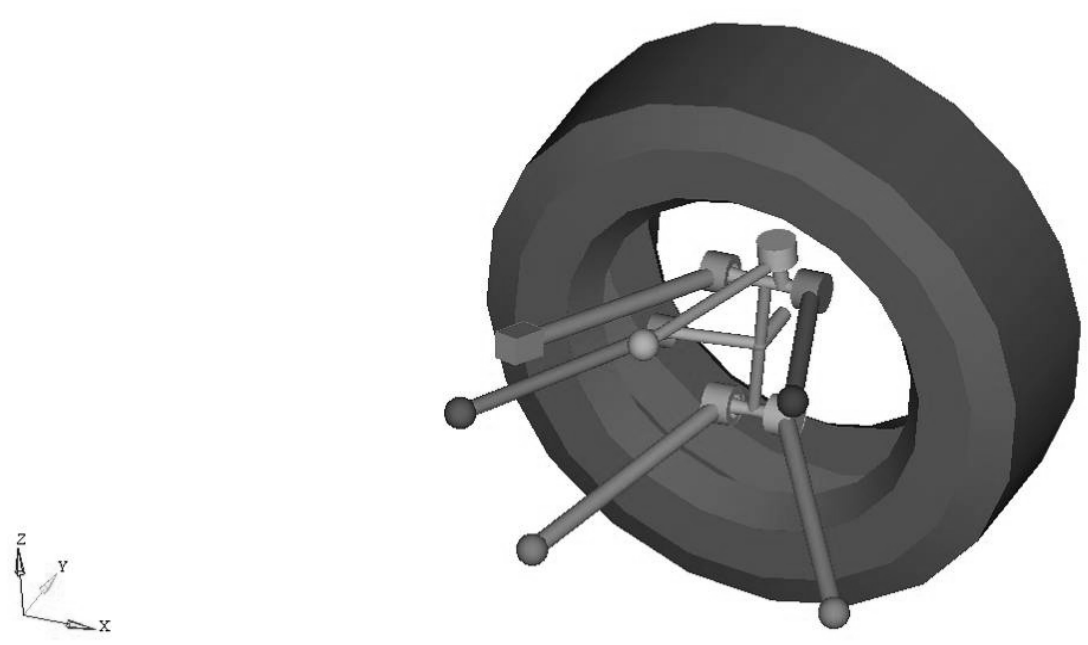

Figure 3: CAD model of suspension (rectangular joint is sliding). 
The example of suspension with two controlled shifting connections is described in Figure 2 and Figure 3, in this case one connection (arrow) is "on" and the other "off”.

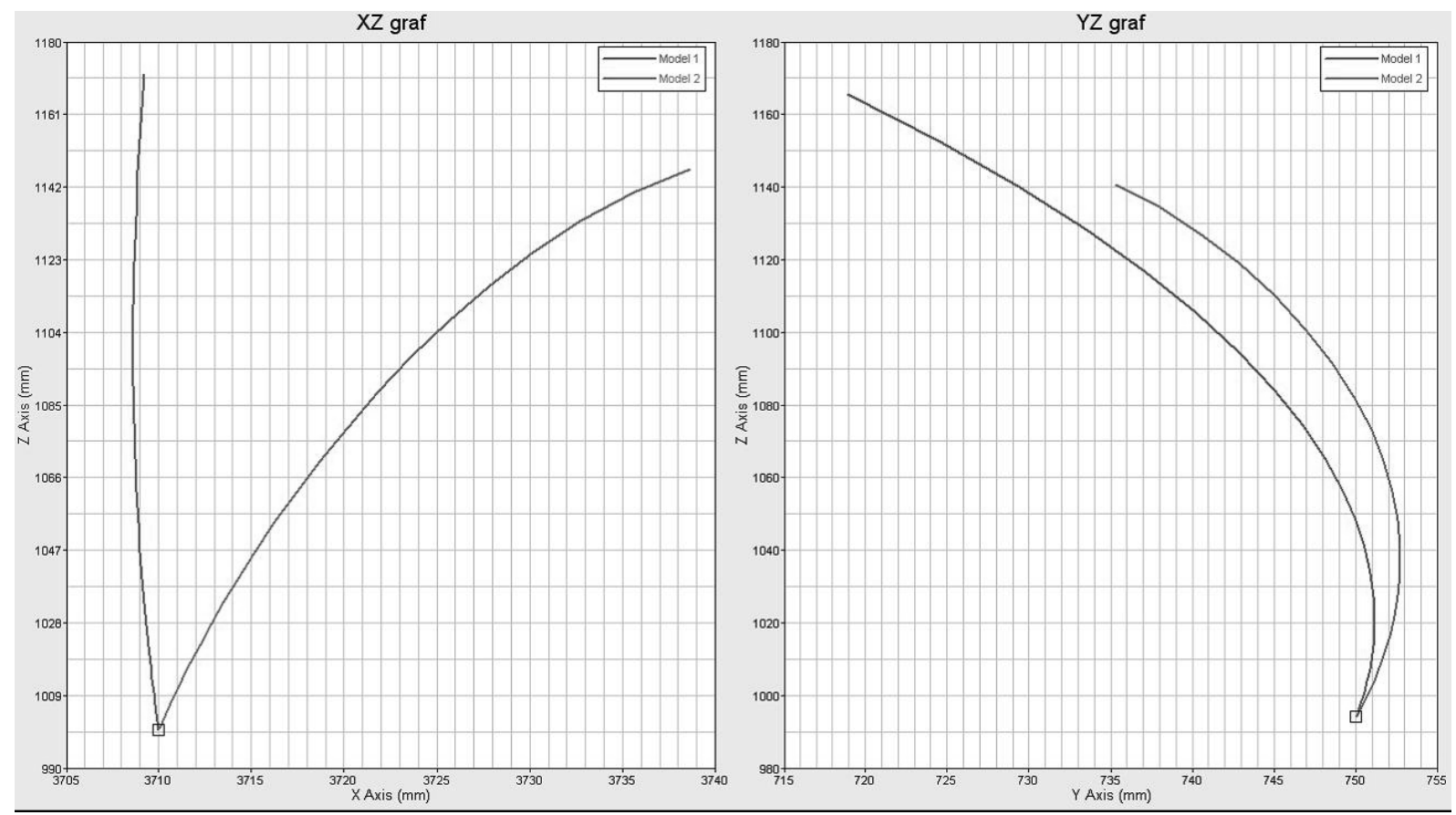

Figure 4: Longitudinal (left) and lateral movement (right graph) of wheel centre point during suspension for two different settings. Suspension movement (in positive $\mathrm{z}$ direction) is on vertical axis, movement starts at a selected low suspension position.

Other characteristics that can be affected in the same way are, for example, the change of wheel camber angle, change of self-steering angle during springing, and the subsequent improvement of wheel-road contact in different situations. The effect of suspension characteristics change can be efficiently used in synergy with the control of systems such as ABS and ESP, eventually ABC, in connection with which it can make up a complex system. The system described has the minimum requirement on the active members and, through that, the energy needed for the control.

\section{CONCLUSION}

The above proposed change of the mechanism can be realized through various design changes, e.g., by the introduction of controlled spring-suspending members in spherical pair joints in the car body. The switching system for these slides can than allow change in kinematic characteristics in such a way as to be advantageous for the respective driving conditions and state. The system described above is a direct contribution to the active safety of driving by means of tyre-road contact and transmission of vibrations from the chassis onto the car body. Even if the proposed solution seems to be promising, further investigations are needed in order to evaluate the engineering feasibility and the reliability of such a suspension system. 


\section{ACKNOWLEDGMENT}

The author is very grateful to Dr. Francesco Frendo, University of Pisa, Italy, for his consultancies and discussions during the evaluation of this concept.

\section{REFERENCES}

Frendo, F., Vitale E.: Elastokinematics analysis of compound crank axle suspension. SAE 09AC-0150, 2009.

Ellis, J. R.: Vehicle Dynamics . London Business Books Limited, 1969.

Mitschke, M.: Dynamik der Kraftfahrzeuge. Springer, 1984.

Schiehlen, W., Valasek, M.: Virtual nonlinear multibody systems, Kluwer Academic Publishers, 2003.

Pauwelussen, J.P., Pacejka, J.B.: Smart vehicles. Sweets and Zeitlinger Publishers, 1995.

Bosch: Driving safety systems. SAE, 1999. 